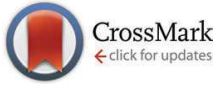

Cite this: Phys. Chem. Chem. Phys., 2015, 17, 28121

Received 16th January 2015, Accepted 4th March 2015

DOI: $10.1039 / \mathrm{c} 5 \mathrm{cp} 00283 d$

www.rsc.org/pccp

\title{
Direct observation of the dealloying process of a platinum-yttrium nanoparticle fuel cell cathode and its oxygenated species during the oxygen reduction reaction $\dagger$
}

\author{
Paolo Malacrida, $\ddagger^{\mathrm{a}}$ Hernan G. Sanchez Casalongue, $\ddagger^{\mathrm{b}}$ Federico Masini, ${ }^{a}$ Sarp Kaya, ${ }^{\mathrm{bc}}$ \\ Patricia Hernández-Fernández, ${ }^{a}$ Davide Deiana, ${ }^{d}$ Hirohito Ogasawara, ${ }^{e}$ \\ Ifan E. L. Stephens, ${ }^{a}$ Anders Nilsson ${ }^{\text {be }}$ and Ib Chorkendorff*a
}

\begin{abstract}
Size-selected $9 \mathrm{~nm} \mathrm{Pt}_{x} \mathrm{Y}$ nanoparticles have recently shown an outstanding catalytic activity for the oxygen reduction reaction, representing a promising cathode catalyst for proton exchange membrane fuel cells (PEMFCs). Studying their electrochemical dealloying is a fundamental step towards the understanding of both their activity and stability. Herein, size-selected $9 \mathrm{~nm} \mathrm{Pt}_{x} \mathrm{Y}$ nanoparticles have been deposited on the cathode side of a PEMFC specifically designed for in situ ambient pressure X-ray photoelectron spectroscopy (APXPS). The dealloying mechanism was followed in situ for the first time. It proceeds through the progressive oxidation of alloyed $Y$ atoms, soon leading to the accumulation of $\mathrm{Y}^{3+}$ cations at the cathode. Acid leaching with sulfuric acid is capable of accelerating the dealloying process and removing these $\mathrm{Y}^{3+}$ cations which might cause long term degradation of the membrane. The use of APXPS under near operating conditions allowed observing the population of oxygenated surface species as a function of the electrochemical potential. Similar to the case of pure Pt nanoparticles, non-hydrated hydroxide plays a key role in the ORR catalytic process.
\end{abstract}

\section{Introduction}

Capable of producing energy through the electrochemical reaction of fuels at near room temperature $\left(<80{ }^{\circ} \mathrm{C}\right)$ operating conditions, proton exchange membrane fuel cells (PEMFCs) are among the most promising renewable energy technologies today. ${ }^{1-4}$ State of the art PEMFC cathodes rely heavily on platinum catalysts to promote the rate of the oxygen reduction reaction (ORR), ${ }^{1,2,5,6}$ but the high catalyst loadings required for adequate performances $-0.5 \mathrm{mg} \mathrm{cm}^{-2}$ of electrode area - make

\footnotetext{
${ }^{a}$ Center for Individual Nanoparticle Functionality (CINF), Department of Physics, Technical University of Denmark (DTU), DK-2800 Lyngby, Denmark. E-mail: ibchork@fysik.dtu.dk

${ }^{b}$ Joint Center for Artificial Photosynthesis (JCAP), Energy Innovation Hub, Lawrence Berkeley National Laboratory, 1 Cyclotron Road, MS 976-JCAP, Berkeley, California 94720, USA

${ }^{c}$ Department of Chemistry, Koc University, Rumelifeneri Yolu, Sariyer, 34450 Istanbul, Turkey

${ }^{d}$ Center for Electron Nanoscopy (CEN), Technical University of Denmark, Kgs Lyngby DK-2800, Denmark

${ }^{e}$ Stanford Synchrotron Radiation Lightsource (SSRL), SLAC National Accelerator Laboratory, 2575 Sand Hill Rd, Menlo Park, California 94025, USA

$\dagger$ Electronic supplementary information (ESI) available. See DOI: 10.1039/ c5cp00283d

\$ These authors contributed equally to this work.
}

the technology difficult to scale up, if not prohibitively expensive. For example, recent estimates calculate that in order for PEMFCs to be commercially viable for automotives, the ORR catalyst activity would need to be improved between a factor of four and a full order of magnitude. ${ }^{4,7,8}$

One of the tools available for improving the catalytic activity is to optimize the bond strength of the ORR intermediates, ${ }^{9,10}$ such as oxygen and oxygenated intermediates, to the catalyst's active sites. Although platinum displays the highest ORR catalytic activity of any pure metal, ${ }^{11}$ it still binds oxygen and the oxygenated intermediates too tightly, limiting the turnover at the active site. Since it is well known that alloying Pt with other metals can vary the bond strength between the catalyst and the ORR intermediates, ${ }^{12,13}$ it stands to reason that finding the right alloy, one that weakens the catalyst-intermediate interaction at the right level ${ }^{11}$ - will lead to higher ORR catalytic activity. At the same time, the utilization of alloys might help to improve the cost effectiveness of a fuel cell by reducing the total Pt concentration in the catalyst.

Alloys of Pt and late transition metals such as $\mathrm{Fe}, \mathrm{Co}, \mathrm{Ni}, \mathrm{Cu}$ have been extensively studied and optimized for more than two decades, resulting in considerable activity enhancements with respect to pure Pt. ${ }^{12-21}$ Under ORR conditions the surface of all these alloys typically undergoes a process known as "dealloying", with the less noble metal dissolving from the outmost atomic layers 
into the acidic electrolyte. This dissolution results in metastable Pt-rich surfaces, known as Pt-skeleton structures: ${ }^{14}$ their ORR activity is typically enhanced with respect to pure Pt due to combinations of ligand and strain effects. ${ }^{22,23}$ However, extended stability tests indicate that further dealloying of the bulk, which occurs on the long term, destabilizes and deactivates these catalysts. ${ }^{24,25}$

Recently, a new class of catalytic materials based on Pt and early transition metal such as Y or Sc, ${ }^{26-29}$ or lanthanide metals such as La, Ce, Gd, also emerged. ${ }^{3,30,31}$ The guiding idea is that the more negative heat of alloy formation of these materials, compared to that of Pt and late transition metals alloys, could prevent dealloying of the bulk, ultimately increasing the stability of Pt-skeleton structures. Experiments on extended surfaces of these alloys have shown great promise in terms of both ORR activity and stability. ${ }^{3,26-31}$ Most notably, size-selected $\mathrm{Pt}_{x} \mathrm{Y}$ nanoparticles have been recently fabricated through a gas aggregation technique. ${ }^{32}$ Despite the novelty of this system and the low level of optimization, $9 \mathrm{~nm} \mathrm{Pt}_{x} \mathrm{Y}$ nanoparticles exhibited an outstanding mass activity (close to the record mass activity of Pt-Ni nanoparticles ${ }^{20}$ ) and moderate stability.

Further improvements to these catalysts could be engendered by more in-depth understanding of the dealloying process. As a matter of fact, the dealloying determines the Pt overlayer formation (i.e. the active phase) but it might also affect the core of the catalyst. It is generally challenging to observe the dealloying of nanoparticles in situ, leaving questions about the exact mechanism. This has been due to the difficulties of probing the system under operating electrochemical conditions with techniques that are at the same time surface sensitive and capable of distinguishing different chemical species. ${ }^{33-35}$ Furthermore, understanding the nature of the adsorbed chemical species involved in the oxygen reduction reaction under operating conditions will lead to further understanding of how to design and optimize future fuel cell catalysts. Both these goals will be addressed through the use of in operando spectroscopy.

Herein, we report on the use of synchrotron-radiation based ambient pressure photoelectron spectroscopy (APXPS), ${ }^{36}$ together with a novel electrochemical cell, ${ }^{37,38}$ to provide in operando measurements of both the dealloying mechanism of a $\mathrm{Pt}_{x} \mathrm{Y}$ nanoparticle electrode surface and the chemical speciation of these nanoparticles during ORR. First, we observe in situ that the dealloying process takes place through the oxidation of yttrium on the nanoparticle surface during progressive operation in the presence of oxygen, leading to the formation of solvated $\mathrm{Y}^{3+}$ cations. We show how the treatment in a liquid acidic electrolyte (e.g. sulfuric acid) might help to further dealloy the catalyst and to remove these $\mathrm{Y}^{3+}$ cations which would eventually degrade the membrane. Second, we identify the oxygenated intermediates of the ORR through their specific $O$ 1s chemical shifts and we observe that non-hydrated hydroxide plays a key role in the ORR catalytic process.

\section{Experimental}

The measurements of this work were performed on size-selected $9 \mathrm{~nm} \mathrm{Pt}_{x} \mathrm{Y}$ nanoparticles which were prepared in a magnetron nanoparticle source ${ }^{39}$ following the same fabrication procedure extensively described previously. ${ }^{32,40}$ This nanoparticle source consists of a magnetron sputter gas aggregation source combined with a time-of-flight mass spectrometer, all assembled into a single ultra-high vacuum (UHV) chamber with a base pressure of $10^{-11}$ mbar. The gas aggregation relies on the $\mathrm{Ar}^{+}$sputtering of a Pt-Y target (Kurt J. Lesker Inc.) with nominal stoichiometry (Pt to $\mathrm{Y}$ atomic ratio) equal to 9. The so formed atomic vapor is condensed into nanoparticles through collisions with cooled $\mathrm{Ar}$ and $\mathrm{He}$ gas. As the nanoparticles produced through this method are typically charged, the time-of-flight mass spectrometer allows to precisely size-select the resulting nanoparticles on the basis of their charge to mass ratio. Following this filtering step, the nanoparticles are directed towards a substrate using Einzel lenses. Depending on the application the substrates consisted of glassy carbon electrodes, for the presented ion scattering spectroscopy (ISS) measurements, of gold grids for TEM applications (300 mesh, Agar Scientific), or of proton exchange membranes (PEM) for the in operando APXPS measurements. The nanoparticle source is also equipped with a number of characterization tools enabling ISS and X-ray photoelectron spectroscopy (XPS). ISS measurements were performed using a $\mathrm{He}^{+}$ion beam with primary ion energy of $1000 \mathrm{eV}$, at a residual He pressure of $5 \times 10^{-8} \mathrm{mbar}$. For the TEM analysis we assumed that morphology and size distribution of the nanoparticles do not vary with different substrates.

The experimental setup for the in operando studies, built on the basis of previous operando electrochemical APXPS works, ${ }^{37,38}$ consisted of a PEEK polymer framework with two chambers separated by a PEM (see Fig. S1 in the ESI $\dagger$ ). The PEM is a Nafion $^{\circledR} 115$ membrane, coated on one side with Pt nanoparticles supported on a Nafion-carbon-black mixture with catalyst loading of $4 \mathrm{mg} \mathrm{cm} \mathrm{cm}^{-2}$ (particle diameter 10-20 nm) from Fuel Cell Store, Inc, Boulder, CO, which serves as the anode of the PEMFC. The blank side of the membrane, which serves as the cathode, was first coated with a thin film of carbon and Nafion through a drop casting method: $0.2 \mathrm{~g}$ Vulcan ${ }^{\circledR}$ XC-72 carbon black and $0.44 \mathrm{~mL}$ $5 \%$ Nafion $^{\circledR}$ solution (Aldrich) were dissolved in approximately $5 \mathrm{~mL}$ of isopropanol and mixed by means of 30 minutes of sonication. $50 \mu \mathrm{L}$ of this mixture was added to a $1 \mathrm{~cm}^{2}$ area of the blank side of the membrane. With the conducting support in place, the membrane was placed in the nanoparticle source, where size selected $9 \mathrm{~nm} \mathrm{Pt}_{x} \mathrm{Y}$ nanoparticles were deposited onto the Nafion-carbon mixture as described above.

After deposition the membranes were vented in air, transported to the APXPS system where the electrochemical cell was assembled. This was then introduced into the APXPS system, where the cathode chamber (the gas cell of the APXPS system) was filled with oxygen gas (or water vapor) and the anode chamber with humidified forming gas $\left(95 \% \mathrm{~N}_{2} / 5 \% \mathrm{H}_{2}\right)$ in order to provide hydrogen fuel and membrane humidification. Both electrodes were connected to an external PineWave potentiostat, allowing the application of potential biases, necessary to study the dealloying process. It should be noticed that the applied potential voltages required to drive the dealloying process are larger than what would be seen in a traditional 3 electrode 
liquid electrochemical setup, mostly due to the lack of a reference electrode in the setup, which means that this voltage scale includes the additional potential required to perform the hydrogen evolution reaction at the counter electrode, and the high series resistance of the Nafion membranes, which are not fully humidified under these reaction conditions.

In order to study the interaction between the alloy and the liquid electrolyte, the cathode was "washed" twice with sulfuric acid outside of the APXPS system. These "washings" consisted of exposing the cathode surface to a droplet of $0.1 \mathrm{M}$ sulfuric acid for 10 minutes, followed by repeated (3-5 times) rinsing with water droplets.

The XPS measurements were performed by placing the electrochemical cell into the gas cell of the APXPS system on beamline 13-2 at the Stanford Synchrotron Radiation Lightsource (SSRL). Since the overall process of preparation of the membranes was realized with the presented methods for the first time, the nanoparticle deposition was performed about two weeks earlier than the first APXPS measurements, the time needed for ensuring a successful cathode preparation, the transfer to the synchrotron facility and a successful assembly of the electrochemical cell. All XPS and electrochemical measurements were performed at room temperature and their binding energies are referenced to the Fermi level as determined from valence band measurements under the corresponding experimental conditions. Spectral deconvolutions and background subtractions (Shirley backgrounds) were performed using Igor-Pro software.

\section{Results and discussion}

In our previous publication we carried out an extensive physical and electrochemical characterization of the size-selected $9 \mathrm{~nm} \mathrm{Pt}_{x} \mathrm{Y}$ nanoparticles investigated herein, albeit deposited on planar glassy carbon supports. ${ }^{32}$ XPS measurements, taken in situ soon after deposition, confirmed the formation of a Pt-Y alloy with a stoichiometry ( $\mathrm{Pt}$ to $\mathrm{Y}$ atomic ratio) of $3.8 \pm 0.6$. ISS spectra demonstrated the presence of both Pt and Y atoms on the surface of such as-deposited nanoparticles. Particle size-distribution histograms confirmed a particle diameter of $8.7 \pm 0.9$, as determined from TEM. Fig. 1a and b show typical TEM images of these catalysts at different magnifications. The shape of the nanoparticles is spherical and the atomic resolution of Fig. 1b shows that the particles consist of various domains, indicating a polycrystalline structure. As already reported for both extended surfaces $^{27}$ and nanoparticles, ${ }^{32}$ under air exposure the $\mathrm{Y}$ atoms of the alloy tend to oxidize at the surface due to the high reactivity and oxophilicity which is typical of these early transition metals. In Fig. 1c the ISS spectrum of the as-deposited nanoparticles is compared to the one after exposure to air. The Y oxidation occurs through the segregation of $\mathrm{Y}$ atoms to the surface, explaining the $\mathrm{Y}$ enrichment in the ISS spectrum of the air-exposed nanoparticles. The presence of a Pt peak indicates that both Y oxide and Pt still coexist on the surface of these catalysts. Scanning transmission electron microscopy energy-dispersive X-ray spectroscopy (STEM-EDS) was previously employed to study the structural stability of these nanoparticles during ORR activity tests in liquid acidic electrolyte (0.1 M perchloric acid): ${ }^{32}$ EDS elemental maps indicated the formation of a core-shell structure as a result of dealloying, with nanoparticles consisting of a $\sim 1 \mathrm{~nm}$ thick $\mathrm{Pt}$ overlayer on top of a $\mathrm{Pt}_{x} \mathrm{Y}$ core.

For the in operando measurements of the dealloying process and the investigations of the chemical surface species present during the ORR, size-selected $9 \mathrm{~nm} \mathrm{Pt}_{x} \mathrm{Y}$ nanoparticles ${ }^{32}$ were analogously deposited on a Nafion-carbon mixture at the cathode side of a PEM which was then transferred to a previously reported APXPS-compatible fuel cell $\operatorname{setup}^{38}$ (for more details, see the Experimental section). The anode, coated with carbon supported Pt nanoparticles, was constantly exposed to humidified hydrogen gas, while the cathode side of the membrane, coated with the Pt-Y catalysts, could be exposed to oxygen gas from the APXPS gas cell.

The preliminary characterization of the coated PEM, after about two weeks of exposure to air, necessary for the transfer to the APXPS setup, showed that the cathode has inhomogeneous $\mathrm{Pt}$ to $\mathrm{C}$ ratio over the membrane, most likely due to low catalyst loading and the roughness of the high surface area Nafioncarbon substrate. However, the $\mathrm{Pt}$ to $\mathrm{Y}$ ratio of the nanoparticles was rather constant: $5.5 \pm 0.8$. This value is slightly higher than the one measured previously on the as-deposited nanoparticles, ${ }^{32}$ probably due to the different experimental
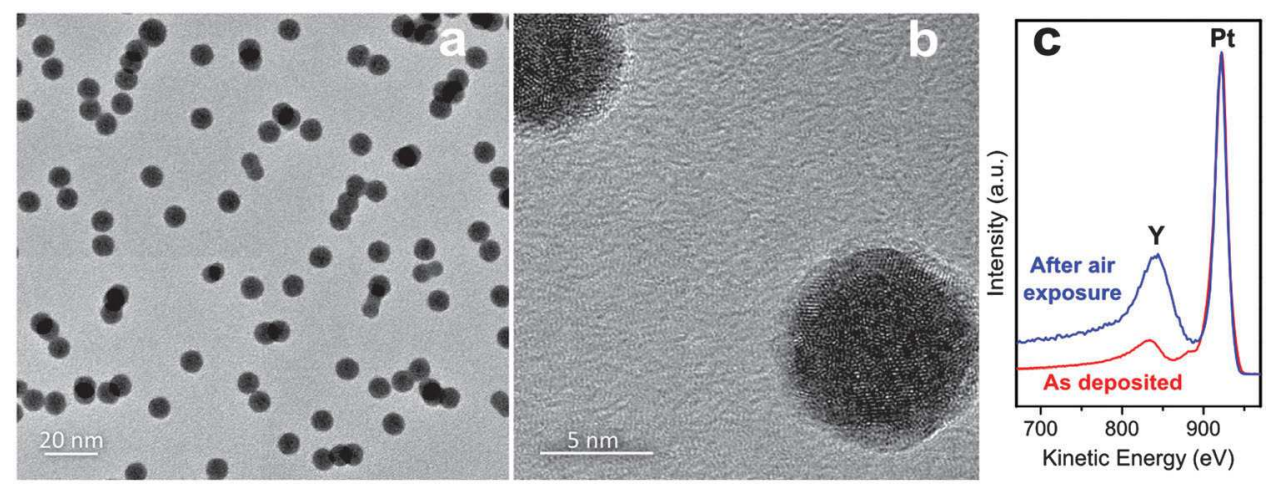

Fig. 1 As-deposited $\mathrm{Pt}_{x} \mathrm{Y}$ nanoparticles characterization. Representative TEM images of size-selected $9 \mathrm{~nm} \mathrm{Pt}_{x} \mathrm{Y}$ nanoparticles at a lower (a) and higher (b) magnification. (c) ISS spectra of $9 \mathrm{~nm} \mathrm{Pt}_{x} \mathrm{Y}$ nanoparticles before and after exposure to air. The peaks at $\sim 920 \mathrm{eV}$ and $\sim 830 \mathrm{eV}$ correspond to Pt and Y respectively. 
conditions (i.e. photon energy, system geometry etc.), the possible effects of air-exposure or the presence of the Nafioncarbon mixture.

Fig. 2a shows the Y 3d XPS spectra of the $\mathrm{Pt}_{x} \mathrm{Y}$ nanoparticle electrode surface probed with an incident photon energy of $700 \mathrm{eV}$ under vacuum (0) and under water vapor pressures of 5 Torr (I-IV) as a function of potential cycling (the system was cycled for 30 minutes between $-0.1 \mathrm{~V}$ and $1.8 \mathrm{~V}$ at a scan rate of $40 \mathrm{mV} \mathrm{s}^{-1}$ ), meant to emulate extended operation in electrochemical environment. The XPS spectra reveal that, as the system is subjected to potential cycling, there is an irreversible change in the surface composition observed by the growth of two higher binding energy features. Fig. $2 \mathrm{~b}$ and $\mathrm{c}$ show a simple peak fitting of the spectra before and after potential cycling. The intensification of the high energy components must be associated with the loss of metallic yttrium (with $\mathrm{Y} 3 \mathrm{~d}_{5 / 2}$ and $\mathrm{Y} 3 \mathrm{~d}_{3 / 2}$ components at 155.9 and $\left.158.0 \mathrm{eV}^{41-44}\right)$ and the formation of an oxidized $\mathrm{Y}$ phase (typical oxidation state of yttrium, for instance in $\mathrm{Y}_{2} \mathrm{O}_{3}: \mathrm{Y}^{3+}$ ) with correspondent $\mathrm{Y} 3 \mathrm{~d}_{5 / 2}$ and $\mathrm{Y} 3 \mathrm{~d}_{3 / 2}$ peaks at 158.7 and $160.8 \mathrm{eV}$.

The assignment of these oxidized components is not trivial and requires some comments. The very high binding energies can only be associated with some oxidized or cationic Y phase, resulting from the reaction of $\mathrm{Y}$ with very electronegative compounds (see Table S1 in the ESI $\dagger$ ). Although there are some reports of Y oxide reaching similarly high binding energies, ${ }^{43,44}$ most authors positioned the $\mathrm{Y}$ components of $\mathrm{Y}_{2} \mathrm{O}_{3}$ at lower energies, with $\mathrm{Y} 3 \mathrm{~d}_{5 / 2}$ lying between 156.4 and $157.2 \mathrm{eV} .^{41,42,45-47}$ Furthermore, the correspondent $\mathrm{O}$ 1s features of $\mathrm{Y}_{2} \mathrm{O}_{3}$ have been all reported between 528.7 and $530.1 \mathrm{eV}$, while for the present sample no significant XPS intensity was observed in this range of binding energies. $\mathrm{Y} 3 \mathrm{~d}_{5 / 2}$ components from $\mathrm{Y}$ hydroxide are expected to appear at higher binding energies compared to $\mathrm{Y}$ oxide and cannot be in principle excluded: however only few literature values have been proposed and these are also lower than those presented in this work. ${ }^{45,47}$ Therefore, we assign these high binding energy components to the presence of $\mathrm{Y}^{3+}$ cations at the cathode side of our PEM. The presence of such cations would be thermodynamically favored ${ }^{48}$ due to the highly acidic conditions induced by Nafion and they would exist either in a solvated form with water molecules or possibly bind to the sulfonic acid groups of Nafion. ${ }^{49}$ As a matter of fact, the high affinity of metal cations such as $\mathrm{Co}^{2+}$ or $\mathrm{Ni}^{2+}$ to these sulfonic groups has been documented previously ${ }^{49-51}$ and it is considered as one important reason of membrane degradation when Pt alloy catalysts are employed in PEMFC. To further support the idea that the $\mathrm{Y}^{3+}$ components of Fig. $2 \mathrm{~b}$ and $\mathrm{c}$ correspond to solvated ions, it should be noted that our previous XPS measurements on air-exposed $\mathrm{Pt}_{x} \mathrm{Y}$ nanoparticles deposited on glassy carbon $^{32}$ (without presence of Nafion) where oxides or hydroxides could be present, allowed to identify oxidized components of $\mathrm{Y}$ at 158.1 and $160.2 \mathrm{eV}$, still significantly lower than those observed here.

A further look at Fig. $2 \mathrm{~b}$ and $\mathrm{c}$ allows noticing that these $\mathrm{Y}^{3+}$ cations are to some extent present in the pristine sample, prior to electrochemical cycling. This is not surprising since the nanoparticles were directly deposited on a Nafion-carbon mixture and subsequently exposed to air for about two weeks: the possible formation of $\mathrm{Y}$ oxide or hydroxide would also lead to the accumulation of $\mathrm{Y}^{3+}$ cations once the membrane is sufficiently humidified due to the highly acidic conditions. Interestingly, the percentage of metallic yttrium of the nanoparticle surface decreases substantially as the nanoparticles are exposed to repeated oxidative and reductive conditions, starting from $35 \%$ of the total Y signal, to less than $20 \%$.

Herein, this oxidation step is monitored under operating conditions while, usually, it is not observed in ex situ measurements. We believe this discrepancy arises from the fact that
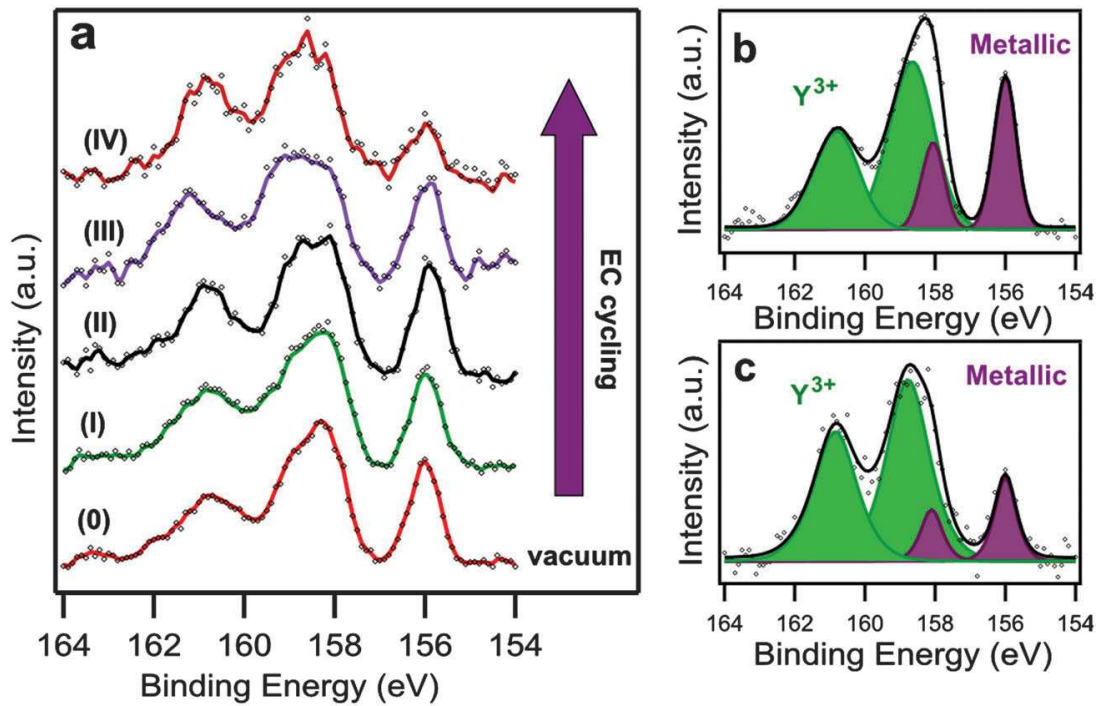

Fig. 2 Dealloying of $\mathrm{Pt}_{X} \mathrm{Y}$ nanoparticles. (a) $\mathrm{Y} 3 \mathrm{~d} X \mathrm{XPS}$ spectra of $\mathrm{Pt}_{x} \mathrm{Y}$ nanoparticles under vacuum (0) and under water vapor pressures of 5 Torr as a function of potential cycling (I-IV) (b) curve fitted $Y$ 3d XPS spectrum before potential cycling. (c) Curve fitted Y $3 d$ XPS spectrum after potential cycling. The incident photon energy was $700 \mathrm{eV}$. 
oxides are readily dissolved and transported away from the catalyst surface in the presence of liquid electrolytes, such as the one utilized in a typical rotating disk electrode (RDE) setup for activity and stability tests on model catalysts (both extended surfaces or nanoparticles). In our in situ setup the absence of any liquid electrolyte would instead limit the mass diffusion and therefore prevent the removal of the $\mathrm{Y}^{3+}$ species, thus providing a unique opportunity to observe the oxidation step of the dealloying mechanism.

In order to verify the diffusion promoted leaching in liquid electrolytes we soaked the cathode of our sample in a sulfuric acid solution. In order to do that, the electrochemical cell was taken out of the APXPS, exposed to sulfuric acid droplets (see Experimental) and reintroduced into the APXPS chamber. As shown in Fig. 3a, the acid induced significant changes on the Y 3d signal, with the deconvolution (shown in Fig. 3b) suggesting the removal of a large part of the $\mathrm{Y}^{3+}$ cations, resulting in more than doubling the relative content of metallic yttrium (from less than $20 \%$ to more than $50 \%$ ). These results support the notion that the dealloying process of the catalyst proceeds through the mechanism shown in Fig. 3c, in which an yttrium oxide/hydroxide intermediate is formed on the catalyst surface during operation, followed by the immediate dissolution of these oxidized species and the release of $\mathrm{Y}^{3+}$ cations into the electrolyte.

The analysis of the Pt to $\mathrm{Y}$ intensity ratios in Fig. $3 \mathrm{~d}$ is in good agreement with this hypothesis. As it was mentioned earlier, the initial $\mathrm{Pt}$ to $\mathrm{Y}$ atomic ratio is equal to $5.5 \pm 0.8$, higher than the one measured on the as-deposited nanoparticles $3.8 \pm 0.6 .^{32}$ This discrepancy seems to be in disagreement with the typical $\mathrm{Y}$ enrichment which is expected as a consequence of air-exposure and Y oxidation (see Fig. 1c) but it is consistent with the formation of $\mathrm{Y}^{3+}$ cations that would dissolve in Nafion leaving a Pt enriched surface. After electrochemical cycling the ratio between Pt and the metallic part of the $\mathrm{Y}$ is highly increased due to $\mathrm{Y}$ oxidation, while the ratio between Pt and the total amount of $\mathrm{Y}$ is unaltered, suggesting that no overall Y loss occurred. Contrarily, after ex situ acid leaching all ratios increase, indicating a substantial removal of Y from the cathode surface. In particular, the increase of the Pt to metallic $\mathrm{Y}$ ratio suggests that the acid treatment was not only able to remove part of the $\mathrm{Y}^{3+}$ cations, but also to accelerate the process of dealloying, leading to a substantial Pt enrichment in the nanoparticles which is in agreement with the formation of a Pt shell, as already reported after RDE experiments in $\mathrm{HClO}_{4}$ solution. ${ }^{32}$

In perspective of a future utilization of $\mathrm{Pt}-\mathrm{Y}$ catalysts into PEMFC for real applications, it will be required to develop leaching protocols for preventing the released $\mathrm{Y}^{3+}$ cations from contaminating and eventually degrading the membrane. ${ }^{4-51}$ The presence of $\mathrm{Y}$ hydroxide/oxide on the surface of the nanoparticles would also represent a problem as it could block Pt active sites and therefore result in lower ORR activity. The reported
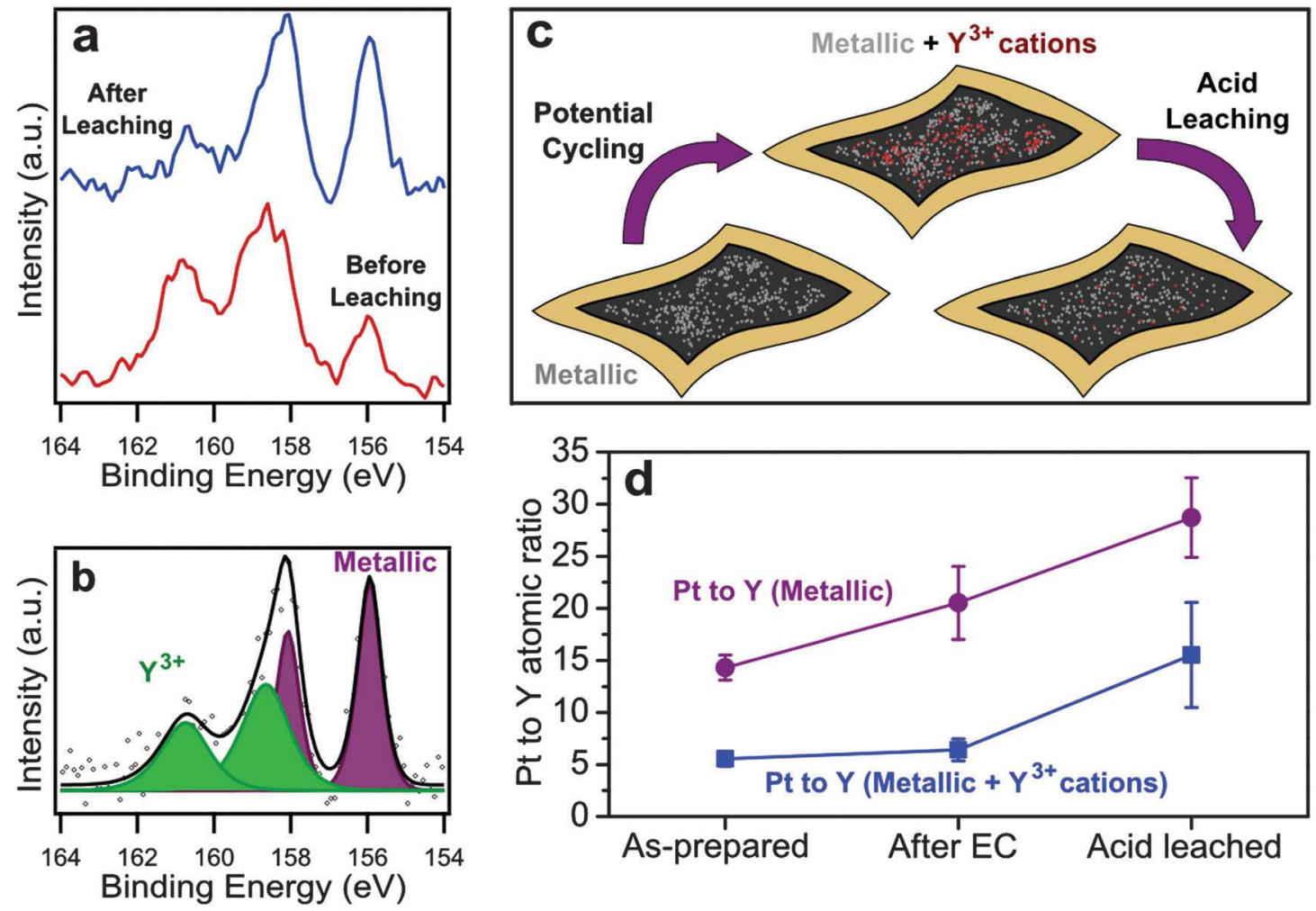

Fig. 3 Acid leaching of $\mathrm{Pt}_{x} \mathrm{Y}$ nanoparticles. (a) $\mathrm{Y} 3 \mathrm{~d}$ XPS spectra of the $\mathrm{Pt}_{x} \mathrm{Y}$ nanoparticles before and after acid leaching. (b) Curve fitted $Y 3 d$ XPS spectrum after acid leaching. The incident photon energy was $700 \mathrm{eV}$. (c) Schematic of the Pt-Y dealloying process. (d) Pt to $Y$ atomic ratios under different experimental conditions. The ratios were determined from the XPS intensities of the Pt $4 f$ and $Y 3 d$ peaks, both considering the metallic component of $Y$ or the total $Y$ intensity. 
leaching protocols typically consist of a treatment in acidic solutions similar to the illustrative acid leaching we conducted in this work. However these methods will need a proper optimization and standardization in order to ensure the complete removal of the $\mathrm{Y}^{3+}$ cations and the formation of a core-shell structure with a pure Pt overlayer on top of an alloyed core, similar to the one achieved after RDE experiments in a liquid electrolyte. ${ }^{32}$ Similar processes, combined with the application of annealing procedures to stabilize ordered Pt overlayers, have been developed for years on Pt-late transition metals alloys, leading to the most active and stable catalysts of this kind. ${ }^{52-54}$

The APXPS fuel cell system also allows probing the chemical species on the cathode surface under near-operating conditions. Fig. 4 shows a series of $\mathrm{O}$ 1s XPS spectra of the pre-leached cathode; they were acquired after the introduction of 2.6 Torr of oxygen gas under constant anodic hydrogen pressure (30 Torr) and open circuit potential (OCP) conditions. The double peak feature in the 536-540 eV range corresponds to the spectral signature of oxygen in gas phase, whose relative shift can be employed to elucidate the potential of the cathode: ${ }^{38,55,56}$ the observed shift of $0.9 \mathrm{eV}$ corresponds to an approximate $900 \mathrm{mV}$ increase in the surface potential as the system evolves towards OCP conditions.

At the right side of Fig. $4(530-532 \mathrm{eV})$ the XPS features can be directly related to oxygenated species bound to the cathode surface, whose binding energies do not vary as a function of the electrochemical potential. ${ }^{38}$ As the surface potential changes, the increase in the XPS intensity of this 530-532 eV region corresponds to the stabilization and growth of oxygenated surface species. ${ }^{38}$ Since these measurements were taken prior to acid treatment, when a considerable amount of oxidized $\mathrm{Y}$ is present, it is important to consider the presence of oxygen containing $\mathrm{Y}$ compounds. Assuming that the oxidized components of the Y $3 d$ XPS spectra are entirely ascribed to $\mathrm{Y}^{3+}$ cations solvated into water layers, these spectra should not contain any traces of $\mathrm{Y}$ oxide/ hydroxide facilitating the study of the oxygenated species present at the cathode. It should be noted that, even in the presence of Y hydroxide which we couldn't a priori exclude, the correspondent $\mathrm{O}$ 1s features in Fig. 4 (according to literature in the range between 531.2 and $531.8 \mathrm{eV}^{45,47}$ ) are not expected to vary or to grow as a function of surface potential due to the extended potential cycling that was already applied to this sample and to the irreversibility of the Y oxidation. Therefore, the qualitative considerations of the following analysis would still be valid.

With the aforementioned assumptions, the modifications in the 530-532 eV region as function of potential are entirely ascribed to oxygenated surface species; by utilizing previously assigned XPS features of various oxygenated adsorbates on Pt as a basis set, it is possible to differentiate six oxygenated species: multilayer water (magenta line) at $533.1 \mathrm{eV}$, adsorbed water $\left(\mathrm{H}_{2} \mathrm{O}_{(\mathrm{ad})}\right.$, orange line) at $532.2 \mathrm{eV}$, adsorbed water hydrogenbonded to hydroxide $\left(\mathrm{H}_{2} \mathrm{O}-\mathrm{OH}\right.$, red line) at $531.6 \mathrm{eV}$, nonhydrated hydroxide $(\mathrm{OH}-\mathrm{O}$, cyan line) at $531 \mathrm{eV}$ and the convolution of hydrated hydroxide $\left(\mathrm{H}_{2} \mathrm{O}-\mathrm{OH}\right)$ and atomic oxygen $\left(\mathrm{O}_{\mathrm{ad}}\right)$ at $530.1 \mathrm{eV}$ (blue line). In agreement with previous results on pure platinum nanoparticles, the surface seems to be dominated by

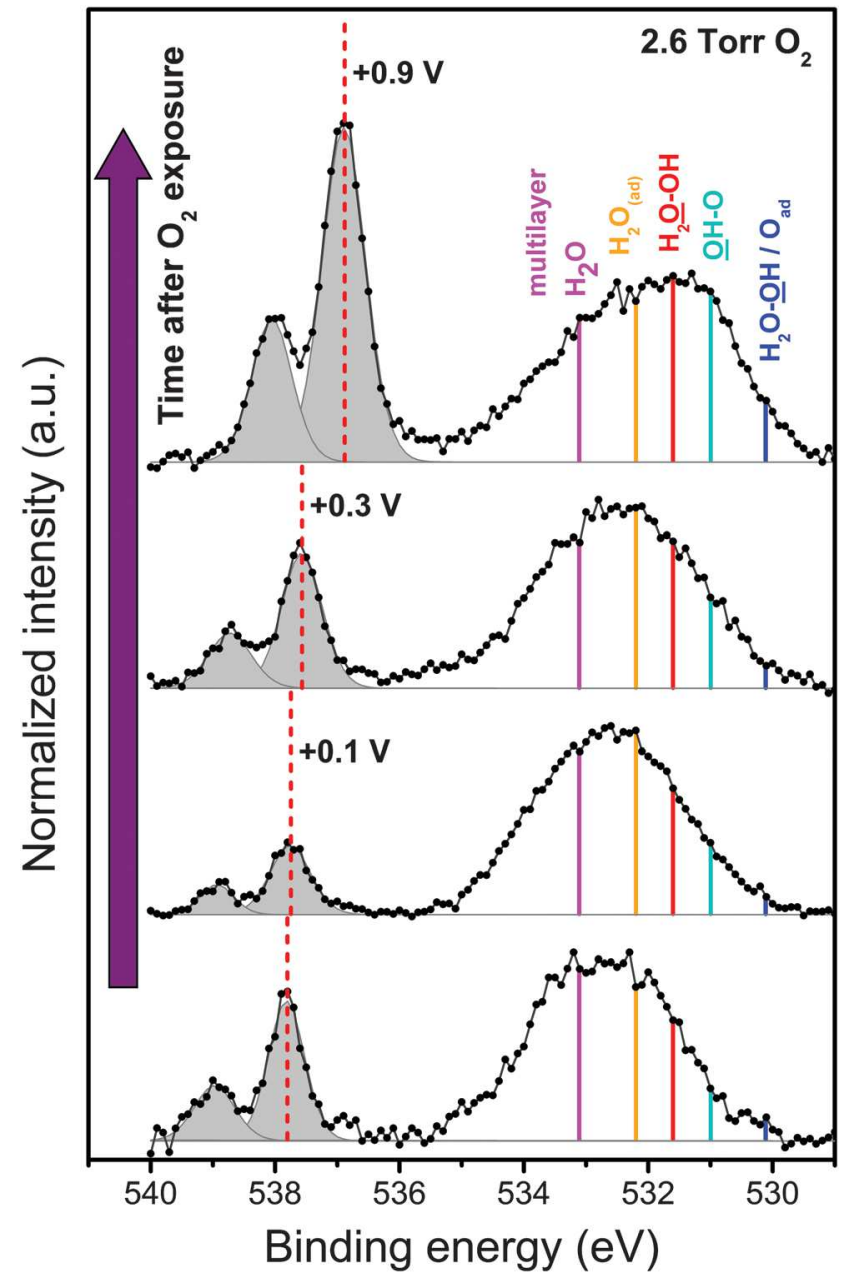

Fig. $4 \mathrm{O}$ 1s XPS spectra of $\mathrm{Pt}_{x} \mathrm{Y}$ nanoparticles under open circuit potential conditions. O 1s XPS spectra after introduction of 2.6 Torr of $\mathrm{O}_{2}$ gas at the cathode while keeping the hydrogen pressure constant at the anode (600 Torr forming gas: $95 \% \mathrm{~N}_{2} / 5 \% \mathrm{H}_{2}$ ). The shift in the double peak feature, corresponding to oxygen gas, can be used to determine the change in surface potential while approaching the equilibrium. The lines in the 529-535 eV region correspond to binding energies of multilayer $\mathrm{H}_{2} \mathrm{O}$ (purple); $\mathrm{H}_{2} \mathrm{O}_{(\mathrm{ad})}$ (orange); $\mathrm{H}_{2} \mathrm{O}-\mathrm{OH}$ (red); $\mathrm{OH}-\mathrm{O}$ (cyan); $\mathrm{H}_{2} \mathrm{O}-\mathrm{OH} / \mathrm{O}_{\text {ad }}$ (blue). The incident photon energy was $700 \mathrm{eV}$.

water at low potentials while, as the system evolves to equilibrium conditions, new oxygenated species begin to populate the surface, with non-hydrated hydroxide being the predominant. Analogous changes in the $\mathrm{O} 1 \mathrm{~s}$ spectra were observed under closed circuit conditions (see Fig. S2 in the ESI $\dagger$ ) while extracting a current from the PEM: in these conditions the surface potential can be tuned by regulating the oxygen pressure at the cathode. As in the case of pure Pt nanoparticles, ${ }^{38}$ the potential dependence of these spectra is similar to that of Fig. 4 indicating the consistency and the reversibility of these measurements. More work will be done to systematically compare the oxygenated species of the alloy catalyst with respect to the pure Pt catalyst. Ideally, the oxygenated species of these Pt-Y cathodes should be studied after application of an optimized acid leaching protocol, allowing to exclude components originating from $\mathrm{Y}$ oxide/hydroxide with certainty. Only in this case and under the same experimental conditions, 
in terms of catalyst loading, membrane humidification and $\mathrm{O}_{2}$ pressures, a quantitative comparison of the oxygenated species will allow a better understanding of the superior catalytic properties of $\mathrm{Pt}_{x} \mathrm{Y}$ nanoparticles for oxygen reduction.

\section{Conclusion}

In conclusion, through the use of APXPS, we were able to follow various steps of the electrochemical dealloying of $\mathrm{Pt}_{x} \mathrm{Y}$ nanoparticles. We observed that this process initially occurs during exposure to air or to the acidic environment resulting from the presence of Nafion that readily leads to the accumulation of $\mathrm{Y}^{3+}$ cations at the cathode side of a PEM. For the first time, it was possible to directly follow the dealloying of the nanoparticles in situ, while the potential was being cycled. Furthermore, we established the important role that liquid acidic electrolytes can play on these catalysts, not only accelerating the nanoparticle dealloying but also improving the diffusion and removal of these $\mathrm{Y}^{3+}$ cations, which compromise the long term stability of the membrane. The result of dealloying and acid leaching is a $\mathrm{Pt}$ enriched surface that helps to maintain the active sites free to catalyze the ORR. The absence of liquid electrolytes at the cathode side of a membrane for PEMFC outlines the necessity of developing an acid leaching protocol for these Pt-Y catalysts. Finally, we have been able to observe that the oxygenated species during ORR resemble those of pure Pt, with non-hydrated hydroxide playing a similarly important role in the ORR catalytic process on Pt-Y alloys.

\section{Acknowledgements}

For funding of this work we gratefully acknowledge the Danish Council for Strategic Research's project NACORR (12-132695) and the Danish National Research Foundation's Center for Individual Nanoparticle Functionality (DNRF54). This work was performed in collaboration with the Joint Center for Artificial Photosynthesis, a Department of Energy (DOE) Energy Innovation Hub: the experimental work was supported by the Joint Center for Artificial Photosynthesis Award DE-SC0004993. Furthermore we gratefully acknowledge the support from Precursory Research for Embryonic Science and Technology (PRESTO), Japan Science and Technology Agency (JST). APXPS experiments were carried out at the Stanford Synchrotron Radiation Lightsource (SSRL), a division of SLAC National Accelerator Laboratory and an Office of Science user facility operated by Stanford University for the U.S. Department of Energy.

\section{References}

1 W. Reitz, Mater. Manuf. Processes, 2007, 22, 789.

2 H. A. Gasteiger and J. Garche, Handbook of Heterogeneous Catalysis, Wiley-VCH Verlag GmbH \& Co. KGaA, 2008.

3 I. E. L. Stephens, A. S. Bondarenko, U. Grønbjerg, J. Rossmeisl and I. Chorkendorff, Energy Environ. Sci., 2012, 5, 6744-6762.
4 H. A. Gasteiger, S. S. Kocha, B. Sompalli and F. T. Wagner, Appl. Catal., B, 2005, 56, 9-35.

5 A. Veziroglu and R. Macario, Int. J. Hydrogen Energy, 2011, 36, 25-43.

6 Y. Wang, K. S. Chen, J. Mishler, S. C. Cho and X. C. Adroher, Appl. Energy, 2011, 88, 981-1007.

7 F. T. Wagner, B. Lakshmanan and M. F. Mathias, J. Phys. Chem. Lett., 2010, 1, 2204-2219.

8 O. Z. Sharaf and M. F. Orhan, Renewable Sustainable Energy Rev., 2014, 32, 810-853.

9 J. Rossmeisl, G. S. Karlberg, T. Jaramillo and J. K. Nørskov, Faraday Discuss., 2009, 140, 337.

10 V. Tripković, E. Skúlason, S. Siahrostami, J. K. Nørskov and J. Rossmeisl, Electrochim. Acta, 2010, 55, 7975-7981.

11 J. K. Nørskov, J. Rossmeisl, A. Logadottir, L. Lindqvist, J. R. Kitchin, T. Bligaard and H. Jónsson, J. Phys. Chem. B, 2004, 108, 17886-17892.

12 V. Stamenković, T. J. Schmidt, P. N. Ross and N. M. Marković, J. Phys. Chem. B, 2002, 106, 11970-11979.

13 V. Stamenkovic, B. S. Mun, K. J. J. Mayrhofer, P. N. Ross, N. M. Markovic, J. Rossmeisl, J. Greeley and J. K. Nørskov, Angew. Chem., 2006, 118, 2963-2967.

14 V. R. Stamenkovic, B. S. Mun, K. J. J. Mayrhofer, P. N. Ross and N. M. Markovic, J. Am. Chem. Soc., 2006, 128, 8813-8819.

15 V. R. Stamenkovic, B. S. Mun, M. Arenz, K. J. J. Mayrhofer, C. A. Lucas, G. Wang, P. N. Ross and N. M. Markovic, Nat. Mater., 2007, 6, 241-247.

16 S. Mukerjee and S. Srinivasan, J. Electroanal. Chem., 1993, 357, 201-224.

17 T. Toda, H. Igarashi, H. Uchida and M. Watanabe, J. Electrochem. Soc., 1999, 146, 3750-3756.

18 S. Koh and P. Strasser, J. Am. Chem. Soc., 2007, 129, 12624-12625.

19 P. Strasser, S. Koh, T. Anniyev, J. Greeley, K. More, C. Yu, Z. Liu, S. Kaya, D. Nordlund, H. Ogasawara, M. F. Toney and A. Nilsson, Nat. Chem., 2010, 2, 454-460.

20 S.-I. Choi, S. Xie, M. Shao, J. H. Odell, N. Lu, H.-C. Peng, L. Protsailo, S. Guerrero, J. Park, X. Xia, J. Wang, M. J. Kim and Y. Xia, Nano Lett., 2013, 13, 3420-3425.

21 T. Anniyev, S. Kaya, S. Rajasekaran, H. Ogasawara, D. Nordlund and A. Nilsson, Angew. Chem., 2012, 124, 7844-7848.

22 J. R. Kitchin, J. K. Nørskov, M. A. Barteau and J. G. Chen, J. Chem. Phys., 2004, 120, 10240-10246.

23 R. R. Adzic, J. Zhang, K. Sasaki, M. B. Vukmirovic, M. Shao, J. X. Wang, A. U. Nilekar, M. Mavrikakis, J. A. Valerio and F. Uribe, Top. Catal., 2007, 46, 249-262.

24 K. J. J. Mayrhofer, K. Hartl, V. Juhart and M. Arenz, J. Am. Chem. Soc., 2009, 131, 16348-16349.

25 S. Chen, H. A. Gasteiger, K. Hayakawa, T. Tada and Y. ShaoHorn, J. Electrochem. Soc., 2010, 157, A82-A97.

26 J. Greeley, I. E. L. Stephens, A. S. Bondarenko, T. P. Johansson, H. A. Hansen, T. F. Jaramillo, J. Rossmeisl, I. Chorkendorff and J. K. Nørskov, Nat. Chem., 2009, 1, 552-556. 
27 I. E. L. Stephens, A. S. Bondarenko, L. Bech and I. Chorkendorff, ChemCatChem, 2012, 4, 341-349.

28 T. P. Johansson, E. T. Ulrikkeholm, P. Hernandez-Fernandez, M. Escudero-Escribano, P. Malacrida, I. E. L. Stephens and I. Chorkendorff, Phys. Chem. Chem. Phys., 2014, 16, 13718-13725.

29 T. P. Johansson, E. T. Ulrikkeholm, P. Hernandez-Fernandez, P. Malacrida, H. A. Hansen, A. S. Bandarenka, J. K. Nørskov, J. Rossmeisl, I. E. L. Stephens and I. Chorkendorff, Top. Catal., 2013, 1-10.

30 M. Escudero-Escribano, A. Verdaguer-Casadevall, P. Malacrida, U. Grønbjerg, B. P. Knudsen, A. K. Jepsen, J. Rossmeisl, I. E. L. Stephens and I. Chorkendorff, J. Am. Chem. Soc., 2012, 134, 16476-16479.

31 P. Malacrida, M. Escudero-Escribano, A. Verdaguer-Casadevall, I. E. L. Stephens and I. Chorkendorff, J. Mater. Chem. A, 2014, 2, 4234-4243.

32 P. Hernandez-Fernandez, F. Masini, D. N. McCarthy, C. E. Strebel, D. Friebel, D. Deiana, P. Malacrida, A. Nierhoff, A. Bodin, A. M. Wise, J. H. Nielsen, T. W. Hansen, A. Nilsson, I. E. L. Stephens and I. Chorkendorff, Nat. Chem., 2014, 6, 732-738.

33 A. S. Bondarenko, I. E. L. Stephens, H. A. Hansen, F. J. Pérez-Alonso, V. Tripkovic, T. P. Johansson, J. Rossmeisl, J. K. Nørskov and I. Chorkendorff, Langmuir, 2011, 27, 2058-2066.

34 M. Wakisaka, H. Suzuki, S. Mitsui, H. Uchida and M. Watanabe, Langmuir, 2009, 25, 1897-1900.

35 M. Watanabe, D. A. Tryk, M. Wakisaka, H. Yano and H. Uchida, Electrochim. Acta, 2012, 84, 187-201.

36 S. Kaya, H. Ogasawara, L.-Å. Näslund, J.-O. Forsell, H. S. Casalongue, D. J. Miller and A. Nilsson, Catal. Today, 2013, 205, 101-105.

37 H. G. Sanchez Casalongue, M. L. Ng, S. Kaya, D. Friebel, H. Ogasawara and A. Nilsson, Angew. Chem., 2014, 126, 7297-7300.

38 H. S. Casalongue, S. Kaya, V. Viswanathan, D. J. Miller, D. Friebel, H. A. Hansen, J. K. Nørskov, A. Nilsson and H. Ogasawara, Nat. Commun., 2013, 4, 2817.

39 R. E. Palmer, S. Pratontep and H.-G. Boyen, Nat. Mater., 2003, 2, 443-448.

40 F. Masini, P. Hernandez-Fernandez, D. Deiana, C. E. Strebel, D. N. McCarthy, A. Bodin, P. Malacrida, I. Stephens and
I. Chorkendorff, Phys. Chem. Chem. Phys., 2014, 16, 26506-26513.

41 Y. Uwamino, T. Ishizuka and H. Yamatera, J. Electron Spectrosc. Relat. Phenom., 1984, 34, 67-78.

42 R. P. Vasquez, M. C. Foote and B. D. Hunt, J. Appl. Phys., 1989, 66, 4866-4877.

43 R. Reichl and K. H. Gaukler, Appl. Surf. Sci., 1986, 26, 196-210.

44 Y. Baba and T. A. Sasaki, Surf. Interface Anal., 1984, 6, 171-173.

45 D. Majumdar and D. Chatterjee, J. Appl. Phys., 1991, 70, 988-992.

46 C. Durand, C. Dubourdieu, C. Vallée, V. Loup, M. Bonvalot, O. Joubert, H. Roussel and O. Renault, J. Appl. Phys., 2004, 96, 1719-1729.

47 R. Xu, S. K. Selvaraj, N. Azimi and C. G. Takoudis, ECS Trans., 2013, 50, 107-116.

48 M. Pourbaix, Atlas of electrochemical equilibria in aqueous solutions, Nat'L Assoc. of Corrosion, [S.l.], 1974.

49 J. Durst, M. Chatenet and F. Maillard, Phys. Chem. Chem. Phys., 2012, 14, 13000-13009.

50 T. Okada, Y. Ayato, M. Yuasa and I. Sekine, J. Phys. Chem. B, 1999, 103, 3315-3322.

51 M. J. Kelly, G. Fafilek, J. O. Besenhard, H. Kronberger and G. E. Nauer, J. Power Sources, 2005, 145, 249-252.

52 K. C. Neyerlin, R. Srivastava, C. Yu and P. Strasser, J. Power Sources, 2009, 186, 261-267.

53 C. Wang, M. Chi, D. Li, D. Strmcnik, D. van der Vliet, G. Wang, V. Komanicky, K.-C. Chang, A. P. Paulikas, D. Tripkovic, J. Pearson, K. L. More, N. M. Markovic and V. R. Stamenkovic, J. Am. Chem. Soc., 2011, 133, 14396-14403.

54 D. Wang, H. L. Xin, R. Hovden, H. Wang, Y. Yu, D. A. Muller, F. J. DiSalvo and H. D. Abruña, Nat. Mater., 2013, 12, 81-87.

55 H. Bluhm, M. Hävecker, A. Knop-Gericke, E. Kleimenov, R. Schlögl, D. Teschner, V. I. Bukhtiyarov, D. F. Ogletree and M. Salmeron, J. Phys. Chem. B, 2004, 108, 14340-14347.

56 C. Zhang, M. E. Grass, A. H. McDaniel, S. C. DeCaluwe, F. E. Gabaly, Z. Liu, K. F. McCarty, R. L. Farrow, M. A. Linne, Z. Hussain, G. S. Jackson, H. Bluhm and B. W. Eichhorn, Nat. Mater., 2010, 9, 944-949. 